G. SONG

KODAI MATH. J.

10 (1987). 285-291

\title{
ON UNIQUE FACTORIZABILITY OF COMPOSITE ENTIRE FUNCTIONS
}

\author{
BY GuODONG SONG
}

\section{Introduction and main results.}

There is a fundamental problem on the factorization theory of entire and meromorphic functions which is so-called "unique factorizability" of composite functions. So far only few results in this topic are known, which are mostly concerned with the unique factorizability theorems for several special types of entire functions (See, e.g., [3], [4]). For example, Ozawa [3] proved the following result.

THEOREM A. Let $g_{0}(z)$ be a prime transcendental entire function of finite order, which has infinitely many zeros. Assume that almost all zeros of $g_{0}(z)$ lie in $\operatorname{Re} Z \geqq x$ for every $x$. Then $g_{0}(z)^{2}$ is uniquely factorizable into two primes.

We shall not state here some basic notions in the factorization theory such as prime, $E$-prime, pseudo-prime, left or right factor, two factorizations of a function being equivalent, etc. One may find the definitions of these notions in references of this paper. However, it seems to be necessary to give some definitions for convenience.

DEFINITION 1. Let $f_{0}$ be a non-linear meromorphic function, $g_{0}$ a non-linear entire function, both are prime. The composite function $F=f_{0}\left(g_{0}\right)$ is called uniquely factorizable if every non-trivial factorization of the form $F=f(g)$ is equivalent to $f_{0}\left(g_{0}\right)$, where $f$ is meromorphic and $g$ entire ( $g$ may be meromorphic when $f$ is rational).

DEFINITION 2. Let $f_{0}$ and $g_{0}$ be two non-linear prime entire functions. The composite function $F=f_{0}\left(g_{0}\right)$ is called $E$-uniquely factorizable if every non-trivial factorization of the form $F=f(g)$ with entire functions $f$ and $g$ is equivalent to $f_{0}\left(g_{0}\right)$.

Our theorem 1 below gives a criterion that under what condition an $E$ uniquely factorizable entire function is uniquely factorizable. It is interesting that the criterion is quite similar to one obtained by Gross [2] concerning the connection between $E$-prime and prime of an entire function.

Received December 16, 1986. 
THEOREM 1. Let $f_{0}$ be a non-linear prime entire function, and $g_{0}$ a prime transcendental entire function. If $f_{0}$ and $F=f_{0}\left(g_{0}\right)$ are both non-periodic, then $F$ is uniquely factorizable if and only if $F$ is E-uniquely factorizable.

The next theorem gives an extension of theorem A.

THEOREM 2. Let $p \geqq 3$ be a prime number. Let $g_{0}(z)$ be a prime non-periodic transcendental entire fuuction, which has at least two different zeros such that there are no $p$ zeros of $g_{0}(z)$ being equally distributed on a circle centred at a zero of $g_{0}(z)$. Then $F(z)=g_{0}(z)^{p}$ is unquely factorizable into two primes.

Remark 1. The following examples

and

$$
F(z)=\left(z \exp z^{p}\right)^{p}=(w \exp p w) \circ z^{p}
$$

$$
G(z)=\left(z\left(z^{p}-1\right) \exp z^{p}\right)^{p}=\left(w(w-1)^{p} \exp p w\right) \circ z^{p}
$$

show that the assumptions about zeros of $g_{0}(z)$ can not be dropped. Here $g_{0}(z)$ $=z \exp z^{p}$ and $\tilde{g}_{0}(z)=z\left(z^{p}-1\right) \exp z^{p}$ are prime (see Song [5, theorem 4]).

We generalize theorem 2 by considering $F(z)=P\left(g_{0}(z)\right)$ with a polynomial $P(w)$ instead of $g_{0}(z)^{p}$, and give the following

THEOREM 3. Let $P(w)$ be a prime (in the sense of composition) polynomial of degree $\geqq 2$, and $g_{0}(z)$ a prime transcendental entire function such that $F(z)=$ $P\left(g_{0}(z)\right)$ is E-right-prime (i.e. every factorization of the form $F=f(g)$ with transcendental entire function $f$ implies that $g$ is linear) and non-periodic. Then $F(z)$ is uniquely factorizable.

Up to now, when we are talking about the unique factorizability, the function $F$ considered has been the composition of two prime functions, $f_{0}$ and $g_{0}$, say. We shall discuss the more general situation when one of $f_{0}$ and $g_{0}$, $f_{0}$ say, is not necessarily prime. In this case we shall first modify the notion "uniquely factorizable" by the following

Definition 3. Let $F(z)$ be a transcendental composite function. $F(z)$ is called left-uniquely factorizable, if there exists a non-trivial factorization $F=f_{0}\left(g_{0}\right)$ with a meromorphic function $f_{0}$ and a prime entire function $g_{0}$ such that every non-trivial factorization of the form $F=f(g)$ implies that either $f(g)$ is equivalent to $f_{0}\left(g_{0}\right)$ or $f$ is a left factor of $f_{0}$.

Similarly, we may define $F(z)$ to be right-uniquely factorizable.

For the special case when $f_{0}(w)$ is $e^{w}$, we obtain the following

THEOREM 4. Let $g_{0}(z)$ be a prime entıre function which is not pseudo-periodic mod any non-zero constant. Then $F(z)=e^{g_{0}(z)}$ is left-uniquely factorizable.

Remark 2. A meromorphic function $f(z)$ is called pseudo-periodic $\bmod \tau$ 
with period $\sigma$ if there are constants $\sigma(\neq 0)$ and $\tau$ such that

$$
f(z+\sigma)-f(z)=\tau \quad \forall z \in \boldsymbol{C} .
$$

Remark 3. The example

$$
F(z)=\exp \left(e^{z}+z\right)=w e^{w} \circ e^{z}
$$

shows that the main assumption of theorem 4 can not be removed. In this example, the prime entire function $g_{0}(z)=e^{z}+z$ is pseudo-periodic $\bmod 2 \pi i$.

\section{Proof of theorem 1 .}

Let $F=f(g)$. We discuss two cases.

(a) $f$ is really meromorphic and $g$ transcendental entire. Then $f(w)$ has exactly one finite pole, $w_{0}$ say, which $g$ doesn't take. Hence

$$
f(w)=\left(w-w_{0}\right)^{-n} f^{*}(w) \text { and } g(z)=w_{0}+e^{M(z)},
$$

where $f^{*}(w)$ is entire with $f^{*}\left(w_{0}\right) \neq 0, n$ is a positive integer, and $M(z)$ is nonconstant entire. Therefore

or

$$
F(z)=e^{-n M(z)} f^{*}\left(w_{0}+e^{M(z)}\right)=\left[e^{-n w} f *\left(w_{0}+e^{w}\right)\right] \cdot M(z),
$$

$$
f_{0} \circ g_{0}(z)=f_{1} \circ M(z),
$$

where $f_{1}(w)=e^{-n w} f^{*}\left(w_{0}+e^{w}\right)$.

Since $F$ is $E$-uniquely factorizable, it follows from (1) that either $M(z)$ is linear which would imply that $F$ is periodic, or $f_{0}\left(g_{0}\right)$ is equivalent to $f_{1}(M)$ which would imply that $f_{0}$ is periodic.

(b) $f(w)$ is really rational and $g(z)$ is really meromorphic. Then $f(w)$ has at most two different poles. We discuss two subcases.

(i) $f(w)=\left(w-w_{0}\right)^{-n}\left(w-w_{1}\right)^{-n_{1}} Q(w)$ with a polynomial $Q$ of degree $\leqq n+n_{1}$ and positive integers $n, n_{1}$. Then

$$
\frac{g(z)-w_{0}}{g(z)-w_{1}}=e^{L(z)}
$$

with an entire function $L(z)$. Put

$$
g *(z)=\frac{g(z)-w_{0}}{g(z)-w_{1}} \equiv \lambda \circ g(z)
$$

and $f^{*}(w)=f \circ \lambda^{-1}(w)$. Then $f \circ g=f^{*} \circ g^{*}$. Hence this case reduces to case (a).

(ii) $f(w)=\left(w-w_{0}\right)^{-n} Q(w)$ with a polynomial $Q$ of degree $\leqq n$ and positive integer $n$. Then

$$
g(z)=w_{0}+\frac{e^{L(z)}}{h(z)}
$$


with entire functions $h(z)$ and $L(z)$. Put

$$
g^{*}(z)=\frac{1}{g(z)-w_{0}} \equiv \lambda \circ g(z)=h(z) e^{-L(z)}
$$

and $f^{*}(w)=f \circ \lambda^{-1}(w)$. Then $f \circ g=f^{*} \circ g^{*}$. Hence this case also reduces to case (a).

q.e.d.

\section{Proof of theorem 2 .}

We shall need the following lemma, which is a corollary to Nevanlinna's second fundamental theorem.

LEMMA 1. Let $f(z)$ be an entire function. Then

$$
\sum_{a \neq \infty}\left(1-\frac{1}{\nu(a)}\right) \leqq 1
$$

where $\nu(a)$ stands for the least order of almost all a-points of $f(z)$. Especially, there is at most one number a such that $\nu(a) \geqq 3$.

In proving theorem 2 we notice first that it suffices to verify $E$-unique factorizability of $F(z)$ by theorem 1 . Let $F=f(g)$ with entire functions $f$ and $g$. We deal with three cases.

(a) $f$ and $g$ are both transcendental. Then, by lemma 1, among zeros of $f(w)$ there is at most one zero with order $n$ such that $(n, p)=1$. Hence 2 cases may occur.

(i) $f(w)=\left(w-w_{0}\right)^{n} h(w)^{p}$ and $g(z)=w_{0}+s(z)^{p}$ with transcendental entire functions $h$ and $s$. Then

or

$$
g_{0}(z)^{p}=s(z)^{n p}\left[h\left(w_{0}+s(z)^{p}\right)\right]^{p}
$$

$$
g_{0}(z)=\eta s(z)^{n} h\left(w_{0}+s(z)^{p}\right) \quad\left(\eta^{p}=1\right) .
$$

But this is impossible, since $g_{0}(z)$ is prime.

$$
f(w)=h(w)^{p}
$$

with an entire function $h$. Then $g_{0}(z)=\eta h(g(z))$. Again impossible.

(b) $f$ is a polynomial. Then we'll obtain (2) or (3) with $h$ being a polynomial. But (2) holds only if $n=1$ and $h$ is a constant, which implies that $f$ is linear. While (3) holds only if $h$ is linear, so that $f(g)$ is equivalent to $g_{0}^{p}$.

(c) $g$ is a polynomial. Then

(i) (2) holds with $s$ being a polynomial, so that $s(z)$ must be linear. Therefore, we may write

$$
g_{0}(z)=k\left(z-z_{0}\right)^{n} h\left(k_{1}\left(z-z_{0}\right)^{p}+w_{0}\right),
$$


where $k$ and $k_{1}$ are constants. By the assumption $g_{0}(z)$ has a zero other than $z_{0}$, hence $h(w)$ has a zero, $w_{1}$ say. Obviously $w_{1} \neq w_{0}$. Now the equation

$$
k_{1}\left(z-z_{0}\right)^{p}+w_{0}=w_{1}
$$

has $p$ roots which are equally distributed on the circle

$$
\left|z-z_{0}\right|=\left|\frac{w_{1}-w_{0}}{k_{1}}\right|^{1 / p}
$$

This violates the assumption.

(ii) (3) holds. We'll deduce that $g(z)$ is linear.

q.e.d.

\section{Proof of theorem 3 .}

In proving theorem 3 two lemmas are needed as follows

LEMMA 2 (Picard's theorem, see [1]). Let $R(u, v)$ be an irreducible polynomial in the 2-dimension complex plane $C[u, v]$. If there are non-constant entire functions $f(z)$ and $g(z)$ such that

$$
R(f(z), g(z))=0, \quad \forall z \in C,
$$

then the Riemann surface defined by $R(u, v)=0$ is of genus 0 .

By a similar method used in [1], the following result can be derived.

LEMMA 3. Let $f(z), g(z)$ be non-constant entire functions, and $P, Q$ nonconstant polynomials. Then the identity

$$
P \circ f(z)=Q \circ g(z), \quad \forall z \in C
$$

implies that there exist rational functions $U, V$ and an entire function $s(z)$ such that

$$
f(z)=U(s(z)), \quad g(z)=V(s(z)) .
$$

Proof. Factorize $P(u)-Q(v)$ into irreducible factors in $C[u, v]$. From (4), we see that one of these irreducible factors, $R(u, v)$ say, satisfies

$$
R(f(z), g(z))=0 \text {. }
$$

By lemma 2, the Riemann surface $X$ defined by $R(u, v)=0$ is of genus 0 . Hence $X$ is conformally equivalent to the Riemann sphere, $S$ say, i.e. its points can be put into $1-1$ correspondence with a parameter $s$ ranging over the Riemann sphere $S$. That is to say that the points $(u, v)$ of $X$ are in $1-1$ correspondence with the points $s$ of $S$ by

$$
u=U(s), \quad v=V(s)
$$


with rational functions $U$ and $V$. By a fractional linear transformation $T$ of $s$ (then $U$ and $V$ are changed into $U\left(T^{-1}\right)$ and $V\left(T^{-1}\right)$, respectively), we may assume that $\mathrm{s}=\infty$ corresponds to a point with $u=\infty$.

Except at a set of finite number of branch points of $X$, which is denoted by $B$, we may use $u$ as local uniformizing parameter, so that $s$ is a holomorphic function of $u, h(u)$ say, near all points of $X$ except those in $B$. Put

$$
s(z)=h(f(z)),
$$

then $s(z)$ is holomorphic near all $z$ except perhaps those for which $(f(z), g(z)) \in B$. These values of $z$ form a discrete set $E$. Clearly, if $z \rightarrow z^{*} \in E$, then $s(z)$ tends to a finite value, which is denoted by $s\left(z^{*}\right)$. Therefore, $z^{*}$ is a removable singularity of $s(z)$. Hence $s(z)$ is entire.

Now on $X u=U(s), v=V(s)$, so that

$$
f(z)=U(s(z)), \quad g(z)=V(s(z)),
$$

and the lemma follows.

Return to the proof of theorem 3. We notice first that by theorem 1 , it suffices to show that $F$ is $E$-uniquely factorizable. Also, since $F$ is $E$-right prime, the only situation to deal with is $F=Q \circ g$ with a polynomial $Q$ of degree $\geqq 2$ and an entire function $g(z)$.

It follows from the equation $P\left(g_{0}\right)=Q(g)$ and lemma 3 that there exist rational functions $U, V$ and an entire function $s(z)$ such that

$$
g_{0}(z)=U(s(z)), \quad g(z)=V(s(z)) .
$$

Since $g_{0}$ is prime, $U$ must be (fractional) linear, and $s(z)=U^{-1}\left(g_{0}(z)\right)$. Thus

$$
P\left(g_{0}(z)\right)=Q\left(V\left(U^{-1}\left(g_{0}(z)\right)\right)\right),
$$

which implies $P=Q\left(V\left(U^{-1}\right)\right)$. Since $P$ is prime, we conclude that $V\left(U^{-1}\right)$ must be linear, so that $Q(g)$ is equivalent to $P\left(g_{0}\right)$. The proof is then complete.

\section{Proof of theorem 4 .}

Let $F=f \circ g$. Three cases will be treated.

(a) $f$ is meromorphic, and $g$ entire, both transcendental.

(i) $f$ has exactly one finite zero or pole, $w_{0}$ say, which $g$ doesn't take, so that

$$
f(w)=\left(w-w_{0}\right)^{n} e^{L(w)} \text { and } g(z)=w_{0}+e^{M(z)},
$$

where $L$ and $M$ are entire, $n \neq 0$ is an integer. Therefore

$$
F(z)=\exp g_{0}(z)=\exp \left\{n M(z)+L\left(w_{0}+e^{M(z)}\right)\right\},
$$

which implies 


$$
g_{0}(z)=\left[n w+L\left(w_{0}+e^{w}\right)+c\right] \cdot M(z) .
$$

Hence $M(z)$ must be linear, and

$$
g_{0}(z)=a z+L\left(w_{0}+e^{b z+c_{1}}\right)+c_{2}
$$

with constants $a(\neq 0), b(\neq 0), c_{1}$ and $c_{2}$. It is evident from (5) that $g_{0}(z)$ is pseudo-periodic with a non-zero constant, which contradicts the assumption.

(ii) $f$ has no zeros or poles. Then $f(w)=e^{L(w)}$ with an entire function $L(w)$. Hence $F(z)=e^{g_{0}(z)}=e^{L(g(z))}$, which implies that $L$ must be linear. Thus $F=f \circ g$ is equivalent to $\exp g_{0}(z)$.

(b) $f$ is rational, and $g$ meromorphic. Then the total number of different poles and zeros of $f$ (including possibly $\infty$ ) is exactly two which $g$ doesn't take, and which may be assumed to be 0 and $\infty$ by a linear transformation. We have $f(w)=c w^{n}$ and $g(z)=e^{M(z)}$ with an entire function $M(z)$ and an integer $n(\neq-1,0,1)$. But $f(w)=c w^{n}$ is a left-factor of $e^{z}$.

(c) $f$ is entire and $g$ is a polynomial. Then $f$ has no zeros, so that $f(w)=e^{h(w)}$, which implies $g_{0}(z)=h(g(z))+c$. Therefore $g(z)$ must be linear.

q. e. d.

\section{REFERENCES}

[1] Fuchs, W. H. J. And Song, Guodong, On a conjecture by M. Ozawa concerning Factorization of entire functions. Ann. Ac. Sci. Fennicae Ser. A. I. Math. Vol. 10, 173-185.

[2] Gross, F., Factorization of entire functions which are periodic mod $g$, Indian J. Pure Appl. Math. 2 (1971), 561-571.

[3] Ozawa, M., On uniquely factorizable entire functions, Kodai Math. Sem. Rep. 29 (1977), 342-360

[4] OzAwA, M., On uniquely factorizable meromorphic functions, Kodai Math. J. 1 (1978), 339-353.

[5] Song, Guodong, On primality of the combination of exponential functions, China Ann. of Math. 6 B(4) (1985), 433-438.

Department of Mathematics

East China Normal University

Shanghai, P.R. China 